\title{
Current and future measurements of semi-inclusive hadron+jet distributions with ALICE
}

\author{
Jaime Norman*, for the ALICE collaboration \\ Laboratoire de Physique Subatomique et Cosmologie, Grenoble, France \\ E-mail: jaime.norman@cern.ch
}

\begin{abstract}
The measurement of jets recoiling from a trigger hadron in heavy-ion collisions can be used to understand the properties of the Quark Gluon Plasma. Jet-medium interactions cause jets to lose energy in the medium and may modify the jet structure. Jet deflection towards large angles due to scattering off of quasi-particles in the Quark-Gluon Plasma may also occur, which can be studied through a measurement of the hadron-jet acoplanarity. These phenomena can be studied through the semi-inclusive distribution of track-based jets recoiling from a trigger hadron. This contribution presents the latest measurements and prospects of semi-inclusive hadron+jet distributions with ALICE. Constraints on energy loss in $\mathrm{p}-\mathrm{Pb}$ collisions and future prospects to measure energy loss in smaller systems are shown. A jet shape measurement of $\mathrm{N}$-subjettiness using recoil jets is outlined. Finally, prospects for hadron+jet acoplanarity measurements with ALICE are presented.
\end{abstract}

International Conference on Hard and Electromagnetic Probes of High-Energy Nuclear Collisions 30 September - 5 October 2018

Aix-Les-Bains, Savoie, France

${ }^{*}$ Speaker. 


\section{Introduction}

Measurements of jets created in ultrarelativistic heavy-ion collisions provide unique probes to characterise the hot and dense QCD medium created in these collisions. The measurement of inclusive jets (see e.g. [1] for recent ALICE results) show a significant suppression in heavy-ion collisions with respect to pp collisions, indicating that partons lose energy while travelling through and interacting with the QCD medium.

The measurement of jets recoiling from a trigger hadron is being employed to further study jet quenching effects. ALICE has measured the trigger-normalised semi-inclusive yield of jets recoiling from a trigger hadron $\frac{1}{N_{\text {trig }}} \frac{\mathrm{d}^{2} N_{\text {jet }}}{\mathrm{d} p_{\mathrm{T}, \mathrm{jet}}} \eta_{\mathrm{jet}}$, differential in jet transverse momentum $p_{\mathrm{T}, \mathrm{jet}}^{\mathrm{ch}}$. A variable $\Delta_{\text {recoil }}$ is then defined as the difference between the trigger-normalised recoil jet distributions in 'reference' and 'signal' trigger track $p_{\mathrm{T}}$ intervals $T T_{\text {ref }}$ and $T T_{\text {sig }}$ [2]:

$$
\Delta_{\text {recoil }}=\left.\frac{1}{N_{\text {trig }}} \frac{\mathrm{d}^{2} N_{\text {jet }}}{\mathrm{d} p_{\mathrm{T}, \text { jet }}^{\text {ch }} \mathrm{d} \eta_{\text {jet }}}\right|_{T T_{\text {sig }}}-\left.c_{\text {ref }} \cdot \frac{1}{N_{\text {trig }}} \frac{\mathrm{d}^{2} N_{\text {jet }}}{\mathrm{d} p_{\mathrm{T}, \mathrm{jet}}^{\text {ch }} \mathrm{d} \eta_{\text {jet }}}\right|_{T T_{\text {ref }}}
$$

where $c_{\text {ref }}$ accounts for the combined effects of invariance of total jet yield with trigger track $p_{\mathrm{T}}$. With this observable one removes entirely the background from uncorrelated reconstructed jets, giving the possibility of extending jet measurements to low- $p_{\mathrm{T}}$ and high jet resolution parameter $R$. The jet population measured with this technique is not biased in terms of jet fragmentation pattern. As a trigger-normalised and semi-inclusive quantity one can also avoid model-dependent assumptions to relate event activity to event geometry, leading to greater systematic sensitivity to jet quenching effects in small systems [3]. It is noted that the measurements shown here use jets reconstructed from charged tracks only, i.e. 'track-based jets'.

\section{Constraints on jet quenching in smaller systems}

The measurement of the trigger-normalised recoil jet distributions in $\mathrm{Pb}-\mathrm{Pb}$ collisions indicates that jets lose a significant amount of energy in $\mathrm{Pb}-\mathrm{Pb}$ collisions, and that this energy is predominantly radiated to angles greater than $R=0.5$ [2]. A similar analysis was performed in $\mathrm{p}-\mathrm{Pb}$ collisions in different event-activity classes, defined using the signal magintude in the V0A detectors of ALICE, to test whether jets are quenched in smaller systems [3]. Figure 1 (left) shows the $\Delta_{\text {recoil }}$ ratio in high/low event activity classes for recoil jets with $R=0.4$ from $15-50 \mathrm{GeV} / c$. The ratio is consistent with unity, indicating minimal jet quenching in $\mathrm{p}-\mathrm{Pb}$ collisions, and a limit of $<0.4 \mathrm{GeV}$ out-of-cone energy loss for jets in this $p_{\mathrm{T}}$ range is set $(90 \% \mathrm{CL}$ ).

The sensitivity to jet quenching in small systems in Run 3/4 of the LHC has also been assessed, based on PYTHIA simulations to estimate the expected number of charged hadron triggers and trigger normalised recoil jet spectrum for a given integrated luminosity. Figure 1 (right) shows the projection of the same observable for $R=0.4$ jets in pp collisions at $\sqrt{s}=14 \mathrm{TeV}$ with an integrated luminosity of 200 pbarn $^{-1}$, where the ratio of 'central' and 'peripheral' events corresponds to the $0-0.1 \%$ centrality percentile and $50-100 \%$ centrality percentile respectively. Here no event-activity shift is included, and the statistical limit (90\% CL) on a measurement of out-of-cone energy loss is $175 \mathrm{MeV}$ for this system. For $\mathrm{p}-\mathrm{Pb}$ collisions where the high-EA is set to the $0-5 \%$ percentile, this 

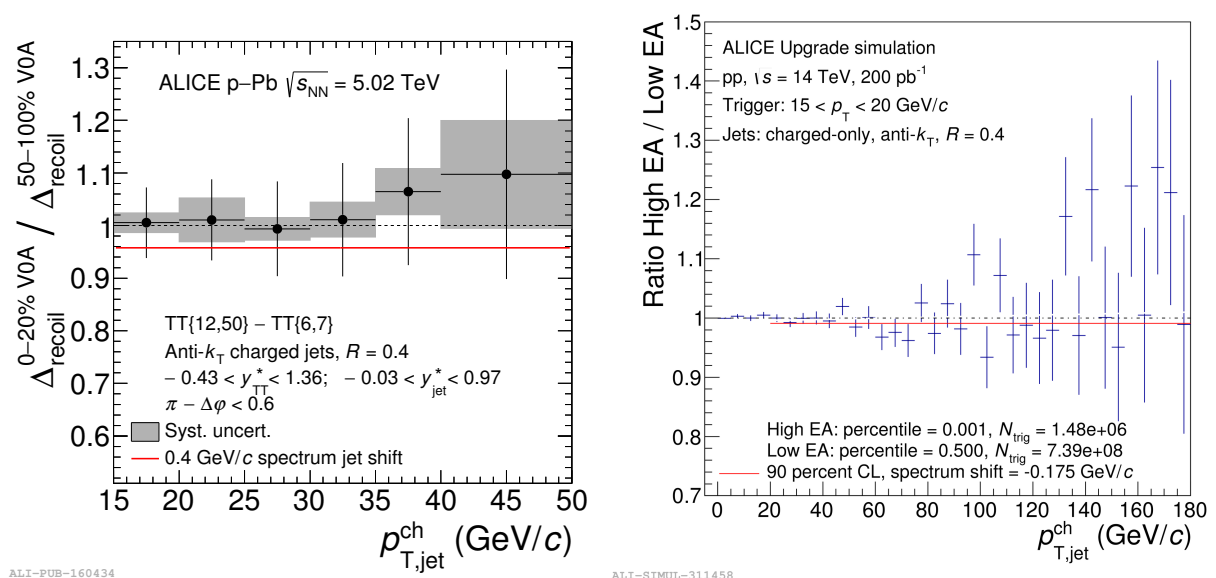

Figure 1: Left: The ratio of $\Delta_{\text {recoil }}$ in high/low event activity classes in $\mathrm{p}-\mathrm{Pb}$ collision at $\sqrt{s_{\mathrm{NN}}}=$ $5.02 \mathrm{TeV}$. Right: Projection of the ratio of $\Delta_{\text {recoil }}$ in high/low event activity classes in pp collisions at $\sqrt{s}=14 \mathrm{TeV}$ in Run 3/4 of the LHC. The red line in both figures corresponds to the $90 \% \mathrm{CL}$ spectrum shift.

limit is $70 \mathrm{MeV}$. While the corresponding systematic uncertainties are not estimated, it is noted that the statistical uncertainties were dominant in the Run 1 measurement.

\section{Substructure of recoil jets}

The measurement of jet shapes and their modification in heavy-ion collisions can give further insight into jet quenching mechanisms. For example, the role of colour coherence [4] can be probed by studying how 2-pronged jets are modified in heavy-ion collisions. The N-prongness of jets is measured through the $\mathrm{N}$-subjettiness observable $\tau_{N}$. For this observable, jets are reclustered using an exclusive clustering algorithm, and 'subjet' axes are found by unwinding the last clustering step. $\tau_{N}$ is then defined as

$$
\tau_{N}=\frac{\sum_{i=1}^{N} p_{\mathrm{T}, \mathrm{i}} \min \left(\Delta R_{\mathrm{i}, 1}, \Delta R_{\mathrm{i}, 2}, \ldots, \Delta R_{\mathrm{i}, \mathrm{N}}\right)}{R_{0} \sum_{i=1}^{N}\left(p_{\mathrm{T}, \mathrm{i}}\right)}
$$

where $\Delta R_{\mathrm{i}, \mathrm{j}}$ is the $\phi-\eta$ distance between track $i$ and subjet $j, p_{\mathrm{T}, \mathrm{i}}$ is the $p_{\mathrm{T}}$ of the $i$-th jet constituent and $R_{0}$ is the jet resolution parameter. The ratio $\tau_{2} / \tau_{1}$ is a measure of how 2-pronged a jet is.

In order to get a combinatorial background-free distribution of low- $p_{\mathrm{T}}$ jets with low fragmentation bias, a similar technique as described in Section 1 is used. As shown in figure 2 (left) a 'reference' trigger track recoil jet distribution is subtracted from a 'signal' trigger track distribution to obtain the $\tau_{2} / \tau_{1}$ distribution of true jets, free from fragmentation bias. Figure 2 (right) shows the $\tau_{2} / \tau_{1}$ distribution in $\mathrm{Pb}-\mathrm{Pb}$ collisions in comparison with the same distribution in PYTHIA. No modification of two-prongness with respect to PYTHIA is seen within the experimental uncertainties. Different reclustering algorithms are also explored which are sensitive to different properties of the jet splitting, see [5] for more information. 

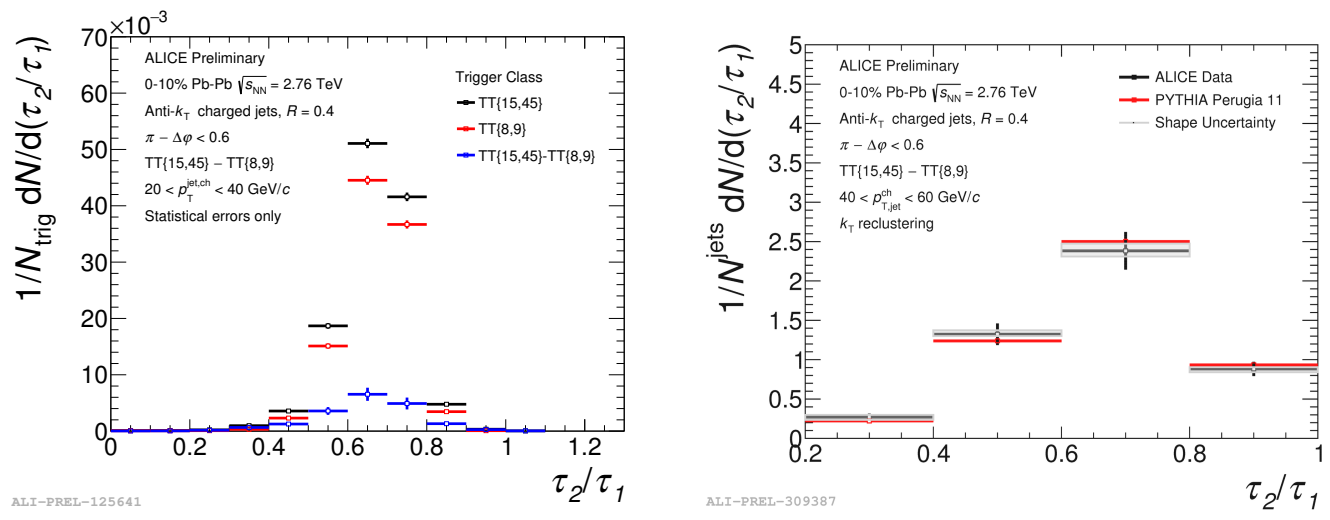

Figure 2: Left: The trigger-normalised $\tau_{2} / \tau_{1}$ distributions in a signal and reference class, and the difference between the two. Right: The trigger-normalised $\tau_{2} / \tau_{1}$ distribution in $\mathrm{Pb}-\mathrm{Pb}$ collisions compared to a PYTHIA reference.

\section{Di-jet azimuthal correlations}

The interaction of jets with the Quark-Gluon Plasma can be further studied by measuring the azimuthal correlation of dijets, or in this case, the azimuthal correlation between a trigger hadron and a corresponding recoiling jet. This is measured by the angle between the trigger hadron and recoiling jet, denoted $\Delta \varphi$. The motivation for studying this observable is two-fold:

1. The broadening of the peak of the away side distribution (at $\Delta \varphi \sim \pi$ ) with respect to vacuum expectation is sensitive to soft multiple scattering in-medium. Since the angular deflection can be related to the change in the momentum transverse to the direction of the initial parton, this could give direct constrains to the transport coefficienct $\hat{q}[6]$.

2. The shape of the tails of the distribution at large angles away from $\pi$ can be used to study the rate of large angle scattering in the QGP. This can arise from resolving the weak degrees of freedom in the Quark-Gluon Plasma, and evidence of large angle scattering could give evidence of a quasiparticle nature of the plasma [7].

The background-subtracted hadron+jet azimuthal distribution was measured in $\mathrm{Pb}-\mathrm{Pb}$ collisions at ALICE with Run 1 data [2]. The rate of large angle scattering showed no deviation from the vacuum expectation within the experimental uncertainties, though this measurement was statistically limited. Recent theoretical work (see e.g. $[7,8]$ ) has suggested that low- $p_{\mathrm{T}}$ jets are most sensitive to such effects and additional, higher statistics measurements are underway.

The reach of a hadron+jet measurement in Run 3/4 of the LHC has been assessed. Central $\mathrm{Pb}-\mathrm{Pb}$ and $\mathrm{pp}$ collisions are simulated with the JEWEL model [9]. Figure 3 (left) shows the background-corrected azimuthal distribution of jets recoiling from a high- $p_{\mathrm{T}}$ trigger hadron, with the expected statistics of Run 3/4. The difference between the large-angle recoil jet yield in pp and $\mathrm{Pb}-\mathrm{Pb}$ collisions is then studied by integrating this distribution at large angles away from $\pi$,

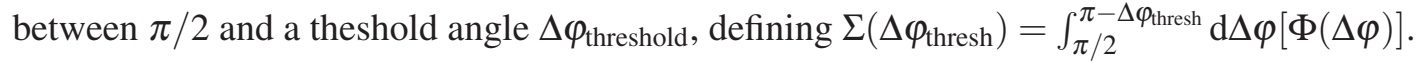


Figure 3 (right) shows the $\Sigma\left(\Delta \varphi_{\text {thresh }}\right)$ distribution in $\mathrm{Pb}-\mathrm{Pb}$ and pp collisions in JEWEL, and the ratio between the two systems. The statistical precision of the ratio at $\Delta \varphi_{\text {threshold }}$ is around $5 \%$ (dominated by the uncertainty of the pp reference), so a deviation in the large-angle yield from vacuum will be able to be resolved to approximately this accuracy. Theoretical calculations predict deviations of similar magnitude [8], so a measurement in Run 3/4 promises to resolve different pictures of the micro-structure of the medium.
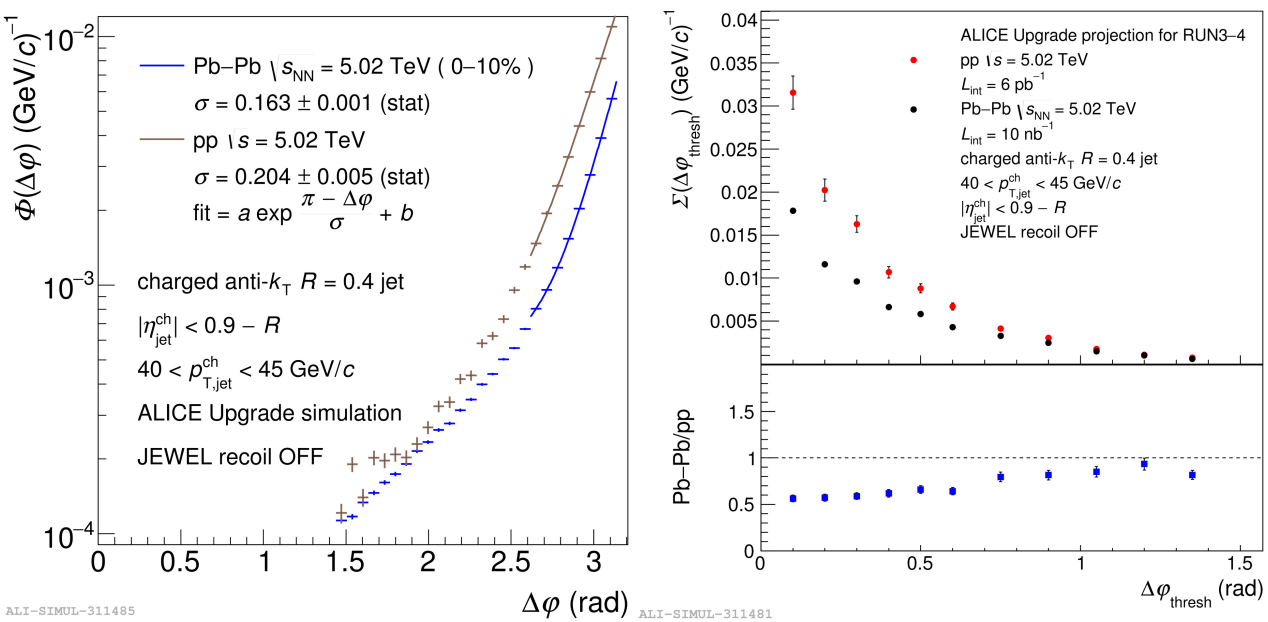

Figure 3: Left: Projection of the background-corrected azimuthal hadron-jet distribution in pp and $\mathrm{Pb}-\mathrm{Pb}$ collisions in Run 3/4. Right: The integral of this distribution at large angles as a function of the threshold angle of integration $\Delta \varphi_{\text {thresh }}$, and its ratio in $\mathrm{Pb}-\mathrm{Pb}$ and $\mathrm{pp}$ collisions.

\section{References}

[1] J. Mulligan, These proceedings, (2018).

[2] ALICE collaboration, J. Adam et al., Measurement of jet quenching with semi-inclusive hadron-jet distributions in central Pb-Pb collisions at $\sqrt{s_{\mathrm{NN}}}=2.76 \mathrm{TeV}$, JHEP 09 (2015) 170, [1506.03984].

[3] ALICE collaboration, S. Acharya et al., Constraints on jet quenching in $p$-Pb collisions at $\sqrt{\mathbf{s}_{\mathbf{N N}}}=$ $5.02 \mathrm{TeV}$ measured by the event-activity dependence of semi-inclusive hadron-jet distributions, Phys. Lett. B783 (2018) 95-113, [1712. 05603$]$.

[4] Y. Mehtar-Tani and K. Tywoniuk, Groomed jets in heavy-ion collisions: sensitivity to medium-induced bremsstrahlung, JHEP 04 (2017) 125, [1610 . 08930].

[5] N. Zardoshti, These proceedings, (2018).

[6] L. Chen, G.-Y. Qin, S.-Y. Wei, B.-W. Xiao and H.-Z. Zhang, Probing Transverse Momentum Broadening via Dihadron and Hadron-jet Angular Correlations in Relativistic Heavy-ion Collisions, Phys. Lett. B773 (2017) 672-676, [1607. 01932].

[7] Y. Yin, These proceedings, (2018).

[8] M. Gyulassy, P. Levai, J. Liao, S. Shi, F. Yuan and X. N. Wang, Precision Dijet Acoplanarity Tomography of the Chromo Structure of Perfect QCD Fluids, 2018. 1808.03238.

[9] K. C. Zapp, JEWEL 2.0.0: directions for use, Eur. Phys. J. C74 (2014) 2762, [1311. 0048 ]. 